\title{
RNA-sequencing analysis reveals transcriptional changes in the roots of low-cadmium-accumulating winter wheat under cadmium stress
}

\author{
Yatao Xiao ${ }^{1,2,3} \cdot$ Zhenjie $^{1{ }^{1,2}} \cdot$ Xuebin $\mathrm{Qi}^{1,2}$ (1) Haiqing $\mathrm{Wu}^{1,2} \cdot$ Wei Guo ${ }^{1,2} \cdot$ Zhijuan Zhao $^{1,2}$
}

Received: 23 November 2017 / Revised: 31 May 2018 / Accepted: 2 January 2019 / Published online: 8 January 2019

(c) The Author(s) 2019

\begin{abstract}
Wheat is the second most important food crop worldwide, and thus, it is important to protect both its yield and quality. Cadmium $(\mathrm{Cd})$ is a heavy metal with strong soil-plant mobility and biological toxicity. Thus, screening low-Cd-accumulating genotypes to elucidate the underlying molecular regulatory mechanisms and gene networks related to Cd stress may be useful to reduce dietary exposure to this heavy metal. Here, the root transcriptomes of the low-Cd-accumulating genotype YM16 treated (YT) or not treated (YC) with Cd were analyzed using the Illumina Hiseq4000 data. The gene expression analysis of YT and YC revealed 1427 differentially expressed genes (DEGs; 429 upregulated and 998 downregulated). Of these, 298 upregulated DEGs were assigned to 41 gene ontology (GO) terms, whereas 537 downregulated DEGs were assigned to $61 \mathrm{GO}$ terms. The pathway enrichment analysis showed that phenylpropanoid biosynthesis, glutathione metabolism, sulfur metabolism, and nitrogen metabolism were significantly enriched in upregulated DEGs, which are involved in defense and detoxification in response to Cd stress. The expression pattern of eight selected DEGs was also analyzed by quantitative real-time polymerase chain reaction, and the results were consistent with those obtained by RNA-sequencing, confirming data reliability. Overall, our study might increase the understanding of complex molecular mechanisms that regulate the responses of plants to $\mathrm{Cd}$ stress, and help develop new improved wheat varieties with low $\mathrm{Cd}$ content in the grain.
\end{abstract}

Keywords Winter wheat $\cdot$ Cadmium $\cdot$ Low-Cd-accumulating genotype $\cdot$ RNA-sequencing

Communicated by G. Klobus.

Yatao Xiao and Zhenjie Du contributed equally to this work and should be considered co-first authors.

Electronic supplementary material The online version of this article (https://doi.org/10.1007/s11738-019-2808-y) contains supplementary material, which is available to authorized users.

Xuebin Qi

qxb6301@sina.cn

1 Farmland Irrigation Research Institute, Chinese Academy of Agricultural Sciences, Xinxiang, Henan 453003, People's Republic of China

2 Key Laboratory of High-efficient and Safe Utilization of Agriculture Water Resources of CAAS, Xinxiang, Henan 453003, People's Republic of China

3 Graduate University of Chinese Academy of Agricultural Sciences, Beijing 100081, People's Republic of China

\section{Introduction}

Cadmium (Cd) is a heavy metal with strong biological toxicity and mobility in the ecosystem (Lima et al. 2006; Ali et al. 2013). Owing to rapid industrial development, high concentrations of $\mathrm{Cd}$ in wastewater, scrap, and exhaust fumes have caused large-scale soil pollution. Controlling $\mathrm{Cd}$ in the food chain is considered essential to protect human health, and consequently, phytoremediation has attracted attention worldwide due to the low repair cost, low soil disturbance, and complete remediation of heavy metals (Ali et al. 2013; Thakur et al. 2016). However, phytoremediation also has some limitations, such as few effective species, selectivity for heavy metal ions, low survival rate of introduced plants, loss of remediation ability by generation advancement, low or no economic value, and slow remediation rate (Ali et al. 2013). Therefore, other methods that will offer short-term solutions are needed to protect food quality.

Previous studies have identified low-Cd-accumulating genotypes of rice (Liu et al. 2005), pepper (Huang et al. 2015a), sweet potato (Huang et al. 2015b), and Chinese 
cabbage (Liu et al. 2010), which can grow in heavily contaminated sites and yield agricultural products with low $\mathrm{Cd}$ levels. Thus, the use of such genotypes is an effective and feasible measure to decrease dietary $\mathrm{Cd}$ exposure (Liu et al. 2005). Cd uptake, accumulation, translocation, and detoxification have been suggested to be genotype-dependent processes (Greger and Löfstedt 2004; Zhang and Duan 2008; Gill et al. 2014). However, the underlying molecular mechanisms remain unclear and might be elucidated by screening low-Cd-accumulating genotypes.

Wheat (Triticum aestivum L.) is the second most important food crop worldwide, and thus, it is crucial to protect both its yield and quality. Direct root-soil contact allows the plant to take up water and nutrients, which is necessary for all physiological and metabolic activities. Therefore, the root is the first organ that senses and responds to biotic and abiotic stresses. Although numerous studies have been conducted to investigate the molecular changes in the roots under abiotic stress conditions, little is known about the stress response mechanisms of low-Cd-accumulating winter wheat genotypes.

Transcriptome studies link genomic information and biological function, which helps to identify key targets of gene regulation related to growth, development, stress adaptation, and evolution (Pandey and Somssich 2009). High-throughput transcriptome techniques have been successfully applied to study different response mechanisms to abiotic stresses, including Cd stress, in many plant species, but not in winter wheat. Therefore, in the present study, root transcriptomic analysis of Cd-treated or untreated low-Cd accumulating winter wheat was carried out to identify the genes and metabolic pathways involved in Cd uptake, translocation and detoxification. Our data might be useful to further explore the molecular mechanisms related to low $\mathrm{Cd}$ accumulation in winter wheat.

\section{Materials and methods}

\section{Plant material and treatments}

In the present study, we used the low-Cd-accumulating genotype Yaomai16 (YM; $0.066 \mathrm{mg} \mathrm{kg}^{-1}$ dry grain weight), which was selected from a field screening trial conducted in Henan Province, China. The screening trial included 79 winter wheat genotypes collected from major wheat-growing areas in China (Henan Province, Hebei Province, Shandong Province, Shanxi Province, and the northern areas of Anhui Province), which were evaluated in Henan Province for 2 consecutive years. The climate in the area is warm, continental, monsoon climate, with an average annual temperature of $14.1^{\circ} \mathrm{C}$, an average annual rainfall of $588.7 \mathrm{~mm}$, a frost-free period of 210 days, and an average annual sunshine duration of $2398.8 \mathrm{~h}$. The physical and chemical properties of the soil are shown in Table $\mathrm{S} 1$. The concentration of $\mathrm{Cd}$ in $\mathrm{YM}$ grains ( $0.066 \mathrm{mg} \mathrm{kg}^{-1}$, dry weight) was significantly lower than that of other genotypes (Fig. S1).

The seeds of YM were immersed in 75\% (v/v) ethanol solution for $5 \mathrm{~min}$ and then rinsed 5 times with deionized water. The soaked seeds were placed on a sterilized gauze for germination at $28{ }^{\circ} \mathrm{C}$. After full emergence, the seeds were transplanted into pots $(38 \times 30 \times 12 \mathrm{~cm})$ containing wellwashed and sterilized sand (particle size of $0.5-0.8 \mathrm{~mm}$ ). At the stage of 2 leaves and 1 bud, 10 seedlings of uniform size were transplanted into a single polyethylene pot filled with 2 L Hoagland's solution ( $\mathrm{pH}$ 6.5), which was replaced every 3 days, and placed in climate-controlled chamber $(12 \mathrm{~h}$ photoperiod $/ 27^{\circ} \mathrm{C}, 12 \mathrm{~h}$ dark period $/ 18^{\circ} \mathrm{C}$; light intensity of $1000 \mu \mathrm{mol} \mathrm{m}{ }^{-2} \mathrm{~s}^{-1}$; Blum and Sullivan 1997). The wall and bottom of the container were covered with black plastic to stimulate root growth. After 10 days, $\mathrm{CdCl}_{2}(100 \mu \mathrm{mol}$ $\mathrm{Cd} \mathrm{L}^{-1}$; YT) was added in the nutrient solution. In YC, no $\mathrm{CdCl}_{2}$ was added. At $48 \mathrm{~h}$ after $\mathrm{Cd}$ exposure (Peng et al. 2015), fresh roots were collected, snap frozen in liquid nitrogen, and stored at $-80{ }^{\circ} \mathrm{C}$ until further analyses. Each treatment was repeated in triplicate.

\section{RNA isolation, library construction, and Illumina sequencing}

Total RNA was isolated from untreated YM roots and those treated with Cd, using the Plant RNA Kit (Omega, USA). The purity of RNA was checked using the NanoPhotometer spectrophotometer (IMPLEN, CA, USA), and the integrity was assessed using the RNA Nano 6000 Assay Kit of the Agilent Bioanalyzer 2100 system (Agilent Technologies, CA, USA). The mRNA was isolated using poly-T oligoattached magnetic beads and fragmented into short segments using the RNA Fragmentation Kit (Ambion). Double-stranded cDNA was synthesized by reverse transcription using random hexamer primers, followed by the addition of buffers, dNTPs, and DNA polymerase I. The cDNA was then purified using AMPure XP beads and modified by end-repair, poly(A) addition, and adaptor ligation. The fragment size of the double-stranded cDNA was selected using AMPure XP beads, and the cDNA library was constructed by polymerase chain reaction (PCR) amplification. cDNA libraries were sequenced using Illumina Hiseq4000 to obtain $150 \mathrm{bp}$ paired-end reads. All the Illumina reads have been submitted to the National Center for Biotechnology Information (http://www.ncbi.nlm.nih.gov/sra) with the accession number SRP125276. 


\section{Read processing and identification of differentially expressed genes}

The original image data files obtained by RNA sequencing (RNA-seq) were transformed into raw reads by CASAVA. Subsequently, the raw reads were filtered to exclude reads containing adaptors, reads with poly-N $(N>10 \%)$, and lowquality reads $(Q \leq 5)$ using in-house perl scripts. The Q20, Q30, and GC content of clean data were calculated to check the quality of reads. The reference genome and gene model annotation files were downloaded from the NCBI (https:// www.ncbi.nlm.nih.gov/genome/11). The reference genome index was built using Bowtie 2.0.6 (Langmead and Salzberg 2012), and paired-end clean reads were aligned to the reference genome using TopHat2 2.1.0 (mismatch parameter, 2; all other parameters, default values) (Kim et al. 2013). The number of reads mapped to each gene was calculated by HTSeq 0.5.4 p3, and the gene expression levels were calculated by the fragments per kilobase of exon model per million mapped reads (FPKM; Mortazavi et al. 2008) method. The aligned reads were analyzed using Cufflinks, and the assembled transcripts were further filtered using the FPKM value $>0$. To test the repeatability and reliability of the results, the relation of expression patterns among YT and YC replicates was tested by Pearson's correlation test. Differential expression analysis (3threebiological replicates per treatment) was performed using the "DESeq" package in $\mathrm{R}$ 1.10.1. The $P$ values were adjusted to control false discovery rate (Benjamini and Hochberg 1995) using DEGsEq. The genes with an adjusted $P$ value of $<0.05$ were assigned as differentially expressed genes (DEGs).

\section{Gene ontology classification and pathway enrichment analysis}

Gene ontology (GO) classification is a standard system that consists of three main ontologies: molecular function, biological process, and cellular component. The GO classification of the DEGs was performed using the "GOseq" package in the $\mathrm{R}$ environment, and the gene length bias was corrected. The GO terms with corrected $P$ values of $<0.05$ were considered significantly enriched. The Kyoto Encyclopedia of Genes and Genomes (KEGG) pathway analysis of DEGs was performed using KOBAS (Mao et al. 2005).

\section{Quantitative real-time PCR analysis}

The total RNA was isolated from YM roots, and the purity and integrity were checked following the method described above. Eight DEGs were selected to validate RNA-seq data by quantitative real-time PCR (q-PCR) using actin as a housekeeping gene. All primers were designed by Invitrogen (Carlsbad, CA, USA) and are listed in Table S2. The
cDNA was prepared using the PrimeScript RT reagent kit with gDNA Eraser (TaKaRa, Tokyo, Japan) according to the instruction of manufacturer. The q-PCR was performed using an optical 96-well plate with the ABI 7500 RealTime System (Applied Biosystems, Foster City, CA, USA). The thermal cycling conditions were as follows: 40 cycles at $95{ }^{\circ} \mathrm{C}$ for $30 \mathrm{~s}, 95^{\circ} \mathrm{C}$ for $5 \mathrm{~s}$, and $60{ }^{\circ} \mathrm{C}$. The relative quantitative method $\left(2^{-\Delta \Delta \mathrm{CT}}\right)$ was used to calculate the fold change in the expression levels of the selected DEGs. All reactions were performed in three technical replicates using one biological sample. For the functional annotation of the eight selected DEGs, their sequences were compared with homologous sequences in the non-redundant (NR) database.

\section{Results}

\section{Transcriptome assembly and quality assessment}

RNA-seq yielded 52,421,235 raw reads for YC and $53,332,260$ raw reads for YT, whereas 51,349,956 and $52,125,145$ clean reads were obtained, respectively, after filtering. The GC content of clean reads was almost identical between YC and YT (Table S3). Of all the clean reads, 93.92\% had a quality score of 20; 62.3-69.39\% were mapped to the wheat reference genome in YT; $53.69-57.41 \%$ were mapped to the wheat reference genome in YC; 48.4-62.47\% were uniquely mapped reads in YT or YC; and 5.3-6.62\% were multiple aligned reads (Table 1).

\section{Analysis of differential transcript expression}

Differential transcript expression was analyzed based on the read count data obtained from the analysis of gene expression levels. The RNA-seq correlation coefficients of FPKM among YC and YT replicates showed that the gene expression patterns were similar (Fig. 1a), confirming the repeatability and reliability of evaluation results. Using FPKM $>1$ as the criterion for gene expression, we found that 45,984 and 43,553 genes were expressed in the roots of YC and YT, respectively. Of these, 41,633 genes were co-expressed in YC and YT, whereas 4351 and 1920 genes were specifically expressed in YT and YC, respectively (Fig. 1b). A total

Table 1 Root transcriptome assembly of Yaomai16 seedlings treated (YT) or not treated (YC) with Cd

\begin{tabular}{lll}
\hline Sample name & YC & YT \\
\hline Total reads & $51,349,956$ & $52,125,145$ \\
Total mapped reads & $28,439,714(55.42 \%)$ & $34,481,400(66.15 \%)$ \\
Multiple mapped reads & $2,860,716(5.57 \%)$ & $3,405,550(6.53 \%)$ \\
Uniquely mapped reads & $25,578,998(49.84 \%)$ & $31,075,850(59.62 \%)$ \\
\hline
\end{tabular}


A

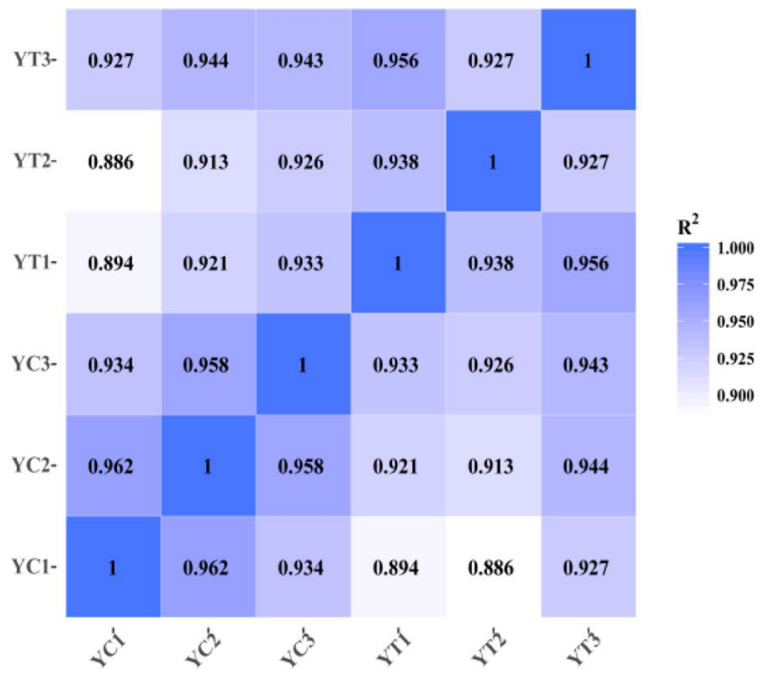

Fig. 1 Root transcriptome analysis of Yaomai16 seedlings treated (YT) or not treated (YC) with Cd. a Correlation coefficients of fragments per kilobase of exon model per million mapped reads among

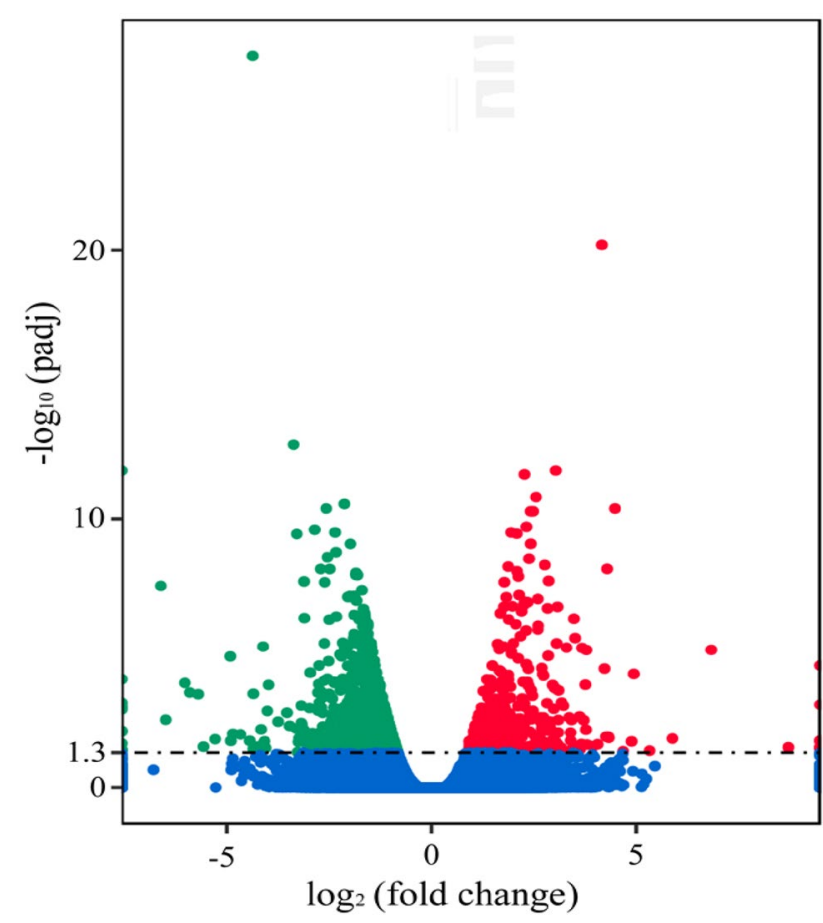

Fig. 2 Differentially expressed genes in the roots of Yaomai16 seedlings treated or not treated with Cd. Red dots, upregulated genes (429); green dots, downregulated genes (998); blue dots, no significant difference $(74,074)$

of 1,427 DEGs (corrected $P$ value of $<0.05$ ) were induced by $C d$ stress, of which 429 were upregulated and 998 were downregulated (Fig. 2).
B

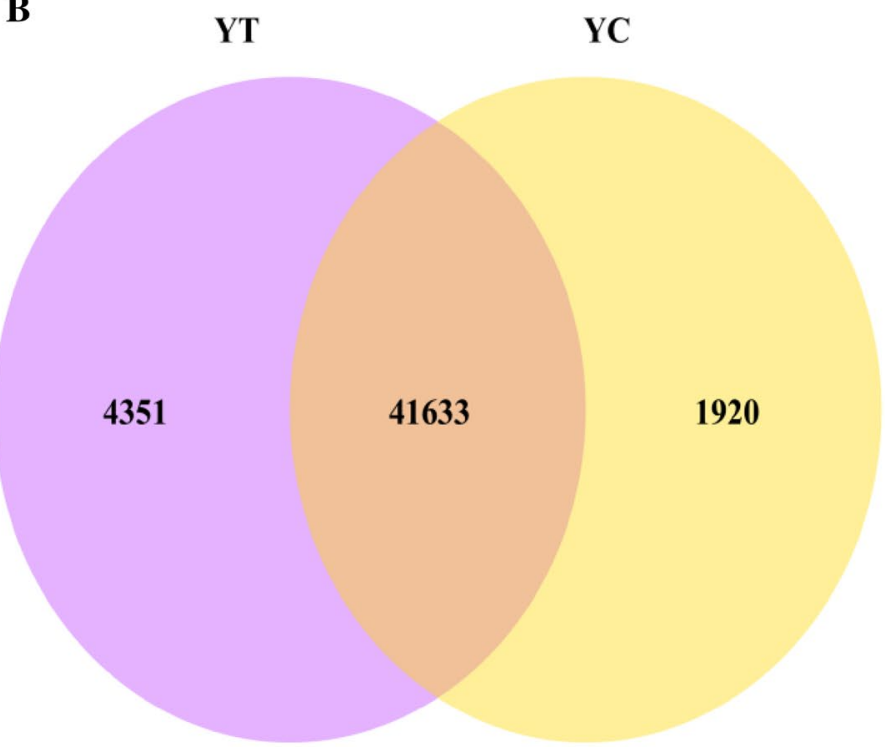

YC and YT replicates. The $R^{2}$ values indicate the correlation coefficients between samples. b Co-expressed and specifically expressed genes in YC and YT root transcriptome

\section{GO enrichment analysis}

The functional annotation and GO enrichment analysis of upregulated and downregulated DEGs showed that 835 DEGs were annotated, of which 298 were upregulated and 537 were downregulated. The upregulated DEGs were assigned to $41 \mathrm{GO}$ terms (biological processes, 21; molecular functions, 19; and cell components, 1). As shown in Fig. 3, the most enriched GO terms of the upregulated DEGs were associated with membrane (GO: 0016020), transport (GO: 0006810), establishment of localization (GO: 0051234), localization (GO: 0051179), response to oxidative stress (GO: 0006979), response to chemical stimulus (GO: 0042221), sulfur compound metabolic process (GO: 0006790), peroxidase activity (GO: 0004601), oxidoreductase activity acting on peroxide as an acceptor (GO: 0016684), antioxidant activity (GO: 0016209), and inorganic anion transmembrane transporter activity (GO: 0015103).

The downregulated DEGs were assigned to $61 \mathrm{GO}$ terms (biological processes, 23; molecular functions, 20; and cell components, 18). As shown in Fig. 4, the most enriched GO terms of the downregulated DEGs were associated with DNA binding (GO: 0003677), membrane-bounded organelle (GO: 0043227), intracellular membrane-bound organelle (GO: 0043231), nucleus (GO: 0005634), protein complex (GO: 0043234), response to stress (GO: 0006950), RNA metabolic process (GO: 0016070), carbohydrate metabolic process (GO: 0005975), RNA biosynthetic process (GO: 0032774), transcription, DNA-dependent (GO: 0006351), response to oxidative stress (GO: 0006979), response to 


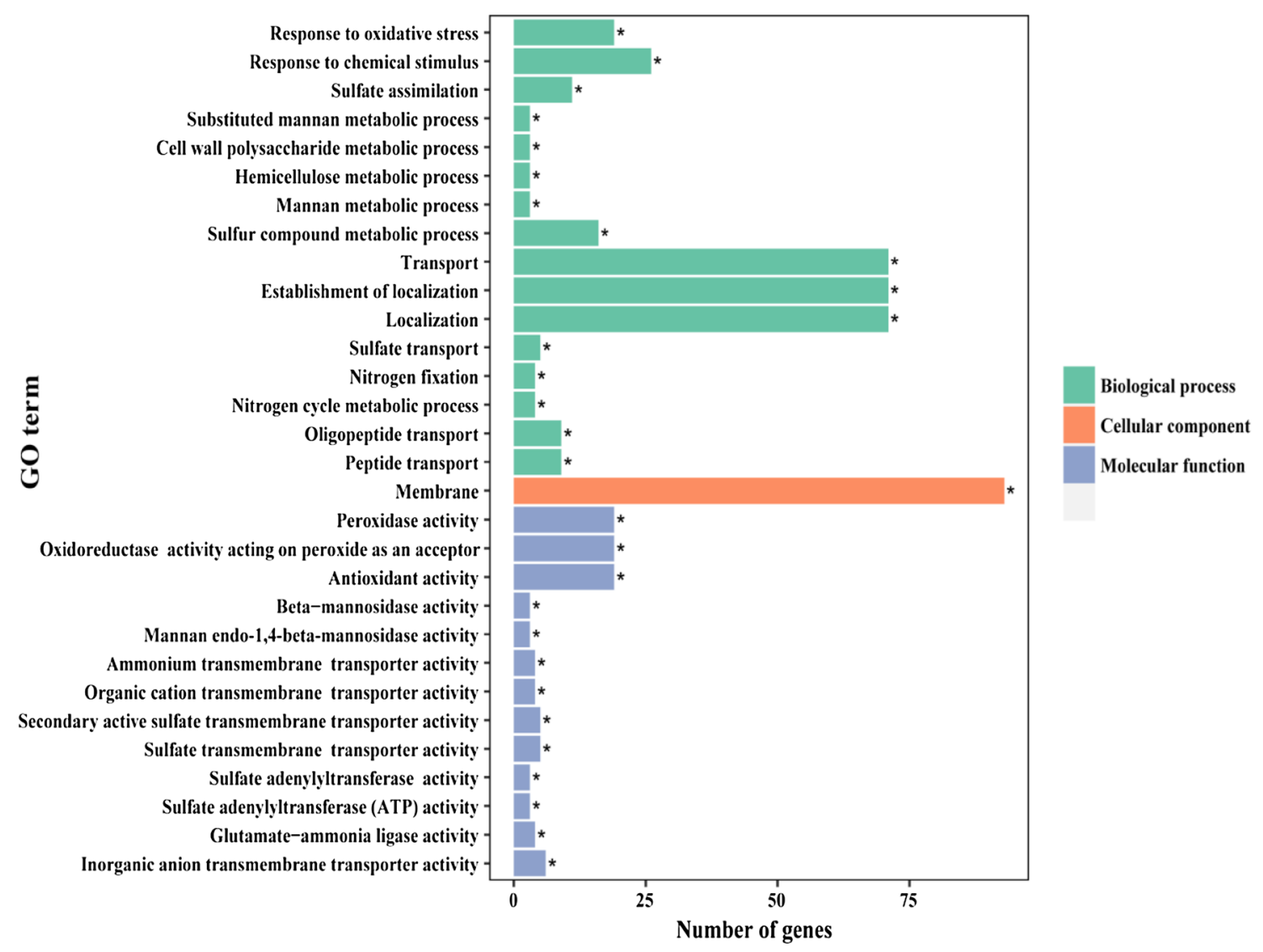

Fig. 3 Gene ontology (GO) classification of the upregulated differentially expressed genes in the root of Yaomai16 seedlings treated with Cd

chemical stimulus (GO: 0042221), and antioxidant activity (GO: 0016209).

\section{KEGG enrichment analysis}

Based on the KEGG enrichment analysis, 923 DEGs were assigned to 90 metabolic pathways, of which biosynthesis of secondary metabolites (194 DEGs; $21.02 \%$ of all DEGs) and metabolic pathways (122 DEGs; $13.22 \%$ of all DEGs) were the most highly enriched, followed by protein processing in the endoplasmic reticulum; phenylpropanoid biosynthesis; glutathione metabolism; galactose metabolism; and valine, leucine, and isoleucine degradation (Fig. 5).

The KEGG enrichment analysis also showed that eight pathways were highly enriched with upregulated DEGs, including phenylpropanoid biosynthesis; glutathione metabolism; sulfur metabolism; ABC transporters; alanine, aspartate, and glutamate metabolism; cysteine and methionine metabolism; nitrogen metabolism; and cyanoamino acid metabolism (Table 2). In addition, five pathways were significantly enriched with downregulated DEGs, including protein processing in the endoplasmic reticulum; phenylpropanoid biosynthesis; valine, leucine, and isoleucine degradation; galactose metabolism; and taurine and hypotaurine metabolism (Table 3). Furthermore, the pathway of phenylpropanoid biosynthesis was significantly enriched in both upregulated and downregulated DEGs (Tables 2, 3).

\section{Validation of RNA-seq by qRT-PCR}

To verify the reliability of RNA-seq, the expression level of eight DEGs (six upregulated and two downregulated DEGs), which might be involved in $\mathrm{Cd}$ stress response, was determined by q-PCR. The gene function was predicted by homologous alignments with sequences in the NR database. The q-PCR showed that the expression pattern of the eight selected DEGs was similar to those found by RNA-seq (Table 4), confirming the reliability of the sequencing data. Furthermore, the functional annotation of the eight selected DEGs was also obtained by comparing with homologous sequences in the non-redundant (NR) database (Table 4). 


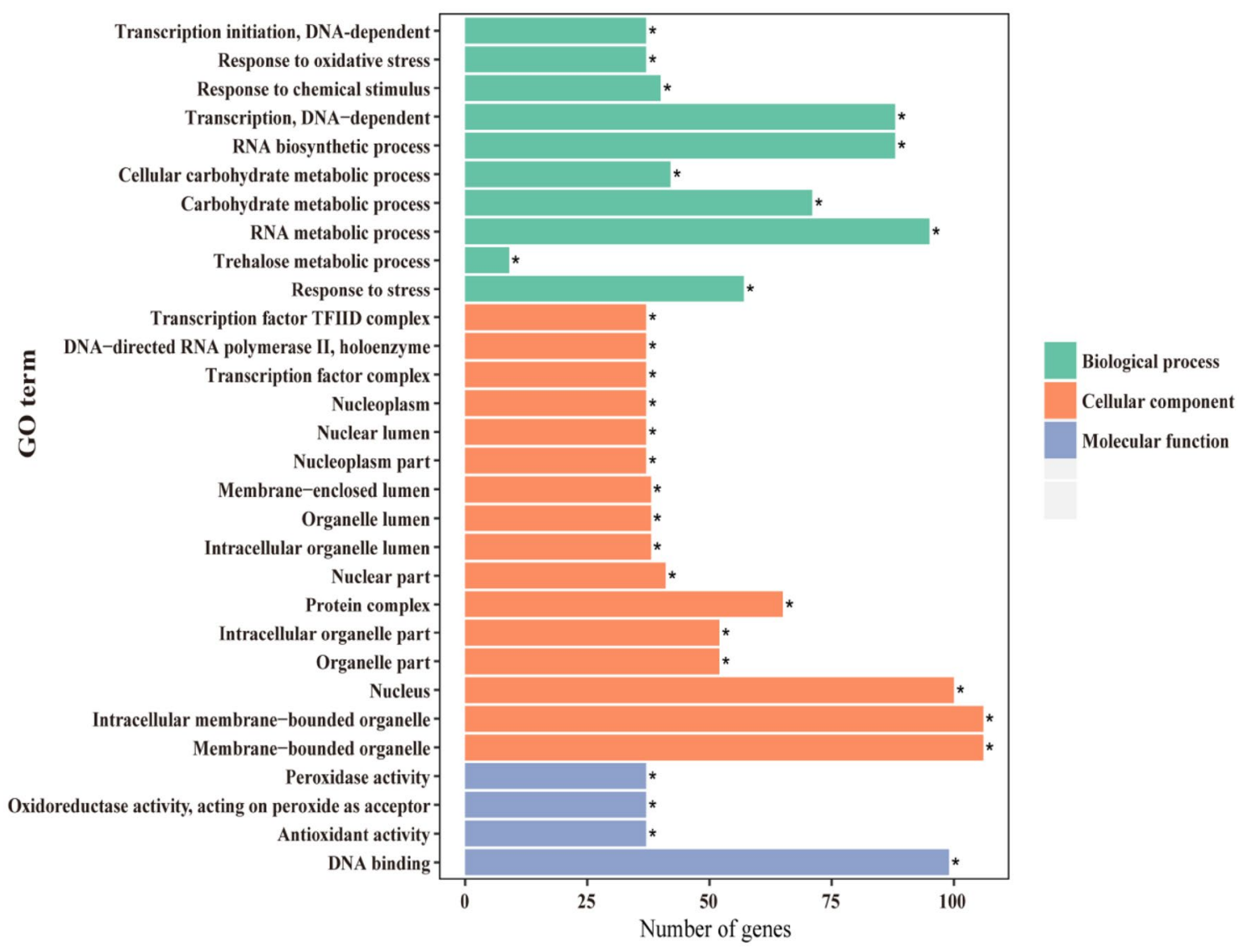

Fig. 4 Gene ontology (GO) classification of the downregulated differentially expressed genes in the root of Yaomai16 seedlings treated with Cd

\section{Discussion}

$\mathrm{Cd}$ is a non-essential element for plants and animals, and its excessive intake can result in irreparable damage (Santos et al. 2016). In vivo, Cd can replace the metal elements (such as $\mathrm{Ca}, \mathrm{Fe}$, and $\mathrm{Zn}$ ) in the activity center of superoxide dismutase, peroxidase, guaiacol peroxidase, ascorbate peroxidase, glutathione reductase, and catalase, which are important protective enzymes against stress, affecting the metabolism of nitrogen, carbohydrate, nucleic acid, and respiration in plant (Assche and Clijsters 1990; Tran and Popova 2013). Even at low concentrations in the soil, $\mathrm{Cd}$ is absorbed by the roots, transported via the xylem and phloem, and accumulated in plant organs (Fujimaki et al. 2010; Fontanili et al. 2016). Cereals are considered as a major source of dietary Cd exposure (Zheng et al. 2007). Therefore, elucidating the underlying mechanisms that regulate plant response to $\mathrm{Cd}$ stress might be helpful to develop genotypes with low $\mathrm{Cd}$ accumulation in the grain.

$\mathrm{Cd}$ as an abiotic stress factor can alter gene expression in plants (Xu 2016). The DEGs are considered useful to discover of potential transcription factors and tolerance genes related to metal transport and signal transduction
(Wang et al. 2013). In the present study, we analyzed the Illumina Hiseq4000 data from the roots of YC and YT to identify the DEGs related to Cd stress. A total of 1,427 DEGs was obtained, of which 429 (30\% of the total DEGs) were upregulated and 998 (70\% of the total DEGs) were downregulated, suggesting that the pattern of gene expression in the root of wheat plant is modified under Cd stress conditions (Fig. 1b). Different genes coordinate their biological functions in organisms (Phillips 2008). Through metabolic pathway enrichment analysis, we can identify the most important biochemical metabolic and signal transduction pathways involved in DEGs. The KEGG pathway enrichment analysis was performed for the upregulated and downregulated DEGs to identify the metabolic pathways that are related to $\mathrm{Cd}$ stress in wheat. The pathways of phenylpropanoid biosynthesis (Pawlak-Sprada et al. 2011), glutathione metabolism (Liu et al. 2016), sulfur metabolism (Liang et al. 2016), and ABC transporters (Song et al. 2014) were significantly enriched in the upregulated DEGs (Table 2), which had been improved to be related with $\mathrm{Cd}$ uptake, translocation, and detoxification. Sulfur metabolism plays a vital role in plant life, regulating the communication between cells and the environment. It improves plant resistance 


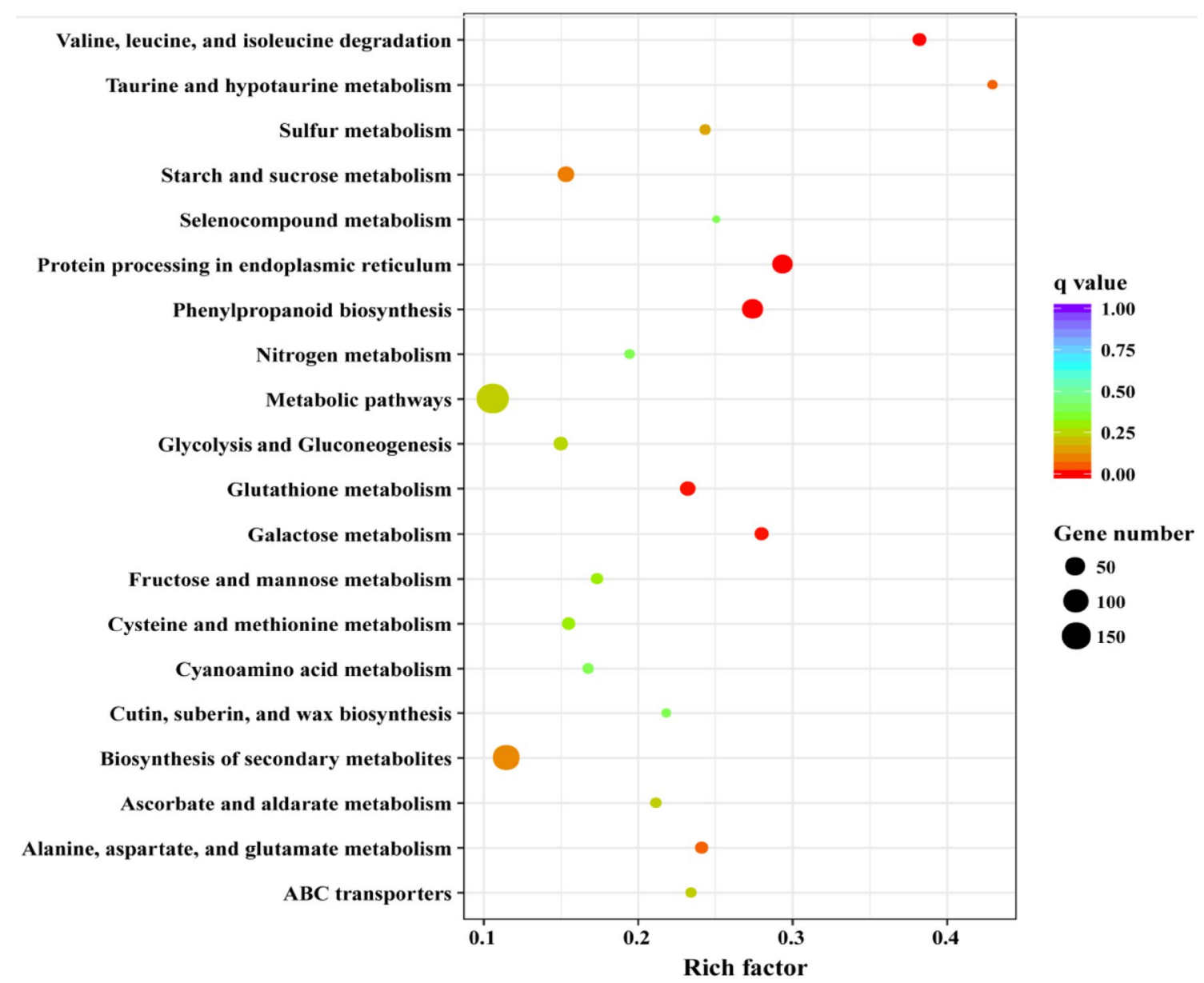

Fig. 5 Most highly enriched pathways in the differentially expressed genes in the root of Yaomai16 seedlings treated with $\mathrm{Cd}$

Table 2 KEGG pathways significantly enriched in the upregulated differentially expressed genes (DEGs) in the root of Yaomai16 seedlings treated with $\mathrm{Cd}$

\begin{tabular}{lllccc}
\hline Pathway ID & Pathway & $\begin{array}{l}\text { Number of DEGs with } \\
\text { pathway annotation }\end{array}$ & $\begin{array}{l}\text { Background } \\
\text { number }\end{array}$ & $P$ value & Corrected $P$ value \\
\hline bdi00940 & Phenylpropanoid biosynthesis & 25 & 224 & $2.96 \mathrm{E}-07$ & $1.63 \mathrm{E}-05$ \\
bdi00480 & Glutathione metabolism & 16 & 104 & $1.06 \mathrm{E}-06$ & $2.92 \mathrm{E}-05$ \\
bdi00920 & Sulfur metabolism & 8 & 33 & $3.47 \mathrm{E}-05$ & 0.000637 \\
bdi02010 & ABC transporters & 7 & 30 & 0.000133 & 0.001829 \\
bdi00250 & Alanine, aspartate, and glutamate metabolism & 8 & 50 & 0.000437 & 0.004807 \\
bdi00270 & Cysteine and methionine metabolism & 9 & 91 & 0.004254 & 0.038997 \\
bdi00910 & Nitrogen metabolism & 5 & 31 & 0.00512 & 0.040226 \\
bdi00460 & Cyanoamino acid metabolism & 6 & 48 & 0.006807 & 0.046799 \\
\hline
\end{tabular}

to Cd stress, as it increases sulfate uptake to enhance the synthesis rate of cysteine, glutathione, and phytochelatins (PCs), thus alleviating Cd toxicity (Rother et al. 2006; Gill et al. 2012; Ferri et al. 2017). In this process, PCs, with glutathione as the precursor, are one of the most important chelating agents in plants (Pereira et al. 2002). It can form non-toxic, low molecular weight $\mathrm{Cd}-\mathrm{S}-\mathrm{PC}$ compounds with $\mathrm{Cd}$, and enter the vacuole through the $\mathrm{Cd} / \mathrm{H}^{+}$reverse transport protein of the vacuolar membrane and the ATP binding cassette $(\mathrm{ABC})$, forming a high-molecular mass compound for storage (Hassan et al. 2005). This not only alleviates the toxicity of $\mathrm{Cd}$, but also blocks the migration of $\mathrm{Cd}$ to 
Table 3 KEGG pathways significantly enriched in the downregulated differentially expressed genes (DEGs) in the roots of Yaomai16 seedlings treated with $\mathrm{Cd}$

\begin{tabular}{lllrrr}
\hline $\begin{array}{l}\text { Pathway } \\
\text { ID }\end{array}$ & Pathway & $\begin{array}{l}\text { Number of DEGs with } \\
\text { pathway annotation }\end{array}$ & $\begin{array}{l}\text { Background } \\
\text { number }\end{array}$ & $P$ value & Corrected $P$ value \\
\hline bdi04141 & Protein processing in the endoplasmic reticulum & 53 & 192 & $1.96 \mathrm{E}-18$ & $1.49 \mathrm{E}-16$ \\
bdi00940 & Phenylpropanoid biosynthesis & 36 & 224 & $2.39 \mathrm{E}-07$ & $9.10 \mathrm{E}-06$ \\
bdi00280 & Valine, leucine, and isoleucine degradation & 13 & 42 & $6.09 \mathrm{E}-06$ & $1.54 \mathrm{E}-04$ \\
bdi00052 & Galactose metabolism & 13 & 61 & 0.000168 & $3.20 \mathrm{E}-03$ \\
bdi00430 & Taurine and hypotaurine metabolism & 5 & 14 & 0.002977 & $4.52 \mathrm{E}-02$ \\
\hline
\end{tabular}

Table 4 Functional annotation and expression level (RNA-seq vs. q-PCR) of the eight selected differentially expressed genes (DEGs) in the root of Yaomai16 seedlings treated with Cd

\begin{tabular}{llr}
\hline Gene & Functional annotation & RNA-seq \\
\hline Traes_2AS_36F44A53A & APRL1_Zea mays adenosine 5'-phosphosulfate reductase-like [Zea mays] & 2.31 \\
Traes_4BS_6EC31E8D4 & Indole-3-acetic acid-amido synthetase GH3.8 [Zea mays] & -2.64 \\
Traes_4DL_469179461 & TPA: IN2-1 protein [Zea mays] & -1.76 \\
Traes_2BL_1AED96909 & Auxin response factor 6 [Zea mays] & 1.11 \\
Traes_2BS_DDCEEE1DE & TPA: hypothetical protein ZEAMMB73_191811 [Zea mays] & 1.65 \\
Traes_5BL_9A790E8CF & Pathogenesis-related protein PRMS [Triticum urartu] & 1.27 \\
Traes_6BL_986D595B9 & PREDICTED: aldehyde dehydrogenase family 3 member F1-like [Setaria italica] & 3.07 \\
TRAES3BF003100040CFD_g & Glutathione-S-transferase 1 [Eragrostis tef] & -0.55 \\
\hline
\end{tabular}

Negative numbers indicate downregulation

other organelles. This complex coping mechanism of $\mathrm{Cd}$ ions in root cells plays an important role in reducing the upward transport of Cd ions or grain accumulation (Sarwar et al. 2015; Ferri et al. 2017). In addition, coordination of S assimilation with $\mathrm{N}$ and $\mathrm{C}$ metabolism also plays a crucial role in abiotic stress tolerance (Gill and Tuteja 2011; Khan et al. 2016). In the present study, the pathways of plant hormone signal transduction, seleno-compound metabolism, protein export, and histidine metabolism were also enriched in the upregulated DEGs, whereas the pathways of endocytosis, glutathione metabolism, peroxisome, phagosome, plant hormone signal transduction, protein processing in the endoplasmic reticulum, and seleno-compound metabolism were enriched in the downregulated DEGs. The genes involved in these processes also play an important role in the normal metabolism of cells. The downregulation of DEGs revealed that $\mathrm{Cd}$ stress might have no significant effect on plant growth during the early stages; however, $\mathrm{Cd}$ exhibited a significant negative effect on plant cell metabolism at the cellular level. On the other hand, eight DEGs from the glutathione metabolism (Traes_4DL_469179461, TRAES3BF003100040CFD_g), sulfur metabolism (Traes_2AS_36F44A53A, Traes_2BS_DDCEEE1DE), plant hormone signal transduction (Traes_4BS_6EC31E8D4, Traes_2BL_1AED96909, Traes_5BL_9A790E8CF) and histidine metabolism (Traes_6BL_986D595B9) were evaluated by q-PCR. Although the detection range and sensitivity of RNA-seq and q-PCR analyses are different, both can detect changes in the expression pattern of DEGs (Table 4). Thus, the reliability of the results is further verified.

In summary, the sequencing of root transcriptome of a low-Cd-accumulating winter wheat genotype enabled the identification of numerous genes and metabolic pathways related to growth, metabolism, and environmental stimulus response, which are involved in plant response to $\mathrm{Cd}$ stress. Our results shed light on the effects of stress due to heavy metal pollution on the growth, development, and metabolism of wheat roots, and showed the complexity of the underlying molecular mechanisms. Further research is needed on the role of key genes that will help improve the effectiveness of phytoremediation through genetic engineering.

\section{Conclusions}

In the present study, we used RNA-seq technology to elucidate the transcriptional response to Cd stress in the roots of a low-Cd-accumulating wheat genotype. We obtained a large volume of high-quality data and transcript information that we used for the DEG identification, GO and KEGG enrichment analysis, and functional annotation. The pathways of phenylpropanoid biosynthesis, glutathione metabolism, 
sulfur metabolism, and nitrogen metabolism were significantly enriched by the DEGs, explaining the low $\mathrm{Cd}$ accumulation in the grain. Overall, our data might be useful to elucidate the underlying molecular mechanisms that regulate the response to $\mathrm{Cd}$ stress in wheat and to develop new improved varieties with low heavy metal content in the grain.

Author contribution statement $\mathrm{YX}$ performed the experiments and drafted the manuscript. ZD and XQ have contributed in the conception and provided some guidance, and HW, WG, and ZZ have contributed in the analysis of the data and further modifications of the manuscript. All authors read and approved the final version of the manuscript.

Acknowledgements The author would like to extend their sincere gratitude to the Agriculture Water and Soil Environment Field Science Research Station, China, for the permission to carry out the research. I would like to acknowledge the financial support for this research from National Natural Science Foundation of China (Grand No. 51679241, 51709265, 51779260), the National Key Research and Development Program of China (2016YFD0800703) and the Central Public-interest Scientific Institution Basal Research Fund (Farmland Irrigation Research Institute, CAAS, FIRI2017-13).

Open Access This article is distributed under the terms of the Creative Commons Attribution 4.0 International License (http://creativeco mmons.org/licenses/by/4.0/), which permits unrestricted use, distribution, and reproduction in any medium, provided you give appropriate credit to the original author(s) and the source, provide a link to the Creative Commons license, and indicate if changes were made.

\section{References}

Ali H, Khan E, Sajad MA (2013) Phytoremediation of heavy metalsconcepts and applications. Chemosphere 91:869-881

Assche FV, Clijsters H (1990) Effects of metals on enzyme activity in plants. Plant Cell Environ 13:195-206

Benjamini Y, Hochberg Y (1995) Controlling the false discovery rate: a practical and powerful approach to multiple testing. J R Stat Soc Ser B (Methodological) 57:289-300

Blum A, Sullivan CY (1997) The effect of plant size on wheat response to agents of drought stress. I. Root drying. Aust J Plant Physiol 24:35-41

Ferri A, Lancilli C, Maghrebi M, Lucchini G, Sacchi GA, Nocito FF (2017) The sulfate supply maximizing Arabidopsis shoot growth is higher under long-than short-term exposure to cadmium. Front Plant Sci 8:854

Fontanili L, Lancilli C, Suzui N, Dendena B, Yin YG, Ferri A, Ishiii S, Kawachi N, Lucchini G, Fujimaki S, Sacchi GA, Nocito FF (2016) Kinetic analysis of zinc/cadmium reciprocal competitions suggests a possible $\mathrm{Zn}$-insensitive pathway for root-to-shoot cadmium translocation in rice. Rice (N Y) 9:16

Fujimaki S, Suzui N, Ishioka NS, Kawachi N, Ito S, Chino M, Nakamura $S$ (2010) Tracing cadmium from culture to spikelet: noninvasive imaging and quantitative characterization of absorption, transport, and accumulation of cadmium in an intact rice plant. Plant Physiol 152:1796-1806

Gill SS, Tuteja N (2011) Cadmium stress tolerance in crop plants. Plant Signal Behav 6:215-222

Gill SS, Khan NA, Tuteja N (2012) Cadmium at high dose perturbs growth, photosynthesis and nitrogen metabolism while at low dose it up regulates sulfur assimilation and antioxidant machinery in garden cress (Lepidium sativum L.). Plant Sci 182:112-120

Gill SS, Khan NA, Tuteja N (2014) Differential cadmium stress tolerance in five Indian mustard (Brassica juncea L.) cultivars. Plant Signal Behav 6:293-300

Greger M, Löfstedt M (2004) Comparison of uptake and distribution of cadmium in different cultivars of bread and durum wheat. Crop Sci 44:501-507

Hassan MJ, Zhang G, Wu F, Wei K, Chen Z (2005) Zinc alleviates growth inhibition and oxidative stress caused by cadmium in rice. J Plant Nutr Soil Sci 168(2):255-261

Huang BF, Xin JL, Dai HW, Liu AQ, Zhou WJ, Yi YM, Liao KB (2015a) Root morphological responses of three hot pepper cultivars to $\mathrm{Cd}$ exposure and their correlations with $\mathrm{Cd}$ accumulation. Environ Sci Pollut Res Int 22:1151-1159

Huang BF, Xin JL, Dai HW, Zhou WJ, Peng LJ (2015b) Identification of low-Cd cultivars of sweet potato (Ipomoea batatas (L.) Lam.) after growing on $\mathrm{Cd}-$-contaminated soil: uptake and partitioning to the edible roots. Environ Sci Pollut Res Int 22:11813-11821

Khan MIR, Iqbal N, Masood A, Mobin M, Anjum NA, Khan NA (2016) Modulation and significance of nitrogen and sulfur metabolism in cadmium challenged plants. Plant Growth Regul 78:1-11

Kim D, Pertea G, Trapnell C, Pimentel H, Kelley R, Salzberg SL (2013) TopHat2: accurate alignment of transcriptomes in the presence of insertions, deletions and gene fusions. Genome Biol 14(4):R36

Langmead B, Salzberg SL (2012) Fast gapped-read alignment with Bowtie 2. Nat Methods 9(4):357-359

Liang TS, Ding H, Wang GD, Kang JQ, Pang HX, Lv JY (2016) Sulfur decreases cadmium translocation and enhances cadmium tolerance by promoting sulfur assimilation and glutathione metabolism in Brassica chinensis L. Ecotoxicol Environ Saf 124:129-137

Lima AIG, Pereira SIA, de Almeida Paula Figueira EM, Caldeira GCN, de Matos Caldeira HDQ (2006) Cadmium detoxification in roots of Pisum sativum seedlings: relationship between toxicity levels, thiol pool alterations and growth. Environ Exp Bot 55:149-162

Liu JG, Zhu QS, Zhang ZJ, Xu JK, Yang JC, Wong MH (2005) Variations in cadmium accumulation among rice cultivars and types and the selection of cultivars for reducing cadmium in the diet. $\mathrm{J}$ Sci Food Agric 85:147-153

Liu WT, Zhou QX, An J, Sun YB, Liu R (2010) Variations in cadmium accumulation among Chinese cabbage cultivars and screening for Cd-safe cultivars. J Hazard Mater 173::737-743

Liu X, Wu FH, Li JX, Chen J, Wang GH, Wang WH, Hu WJ, Gao LJ, Wang ZL, Chen JH, Simon M, Zheng HL (2016) Glutathione homeostasis and $\mathrm{Cd}$ tolerance in the Arabidopsis sultr1; 1-sultr1; 2 double mutant with limiting sulfate supply. Plant Cell Rep 35:397-413

Mao X, Cai T, Olyarchuk JG, Wei L (2005) Automated genome annotation and pathway identification using the KEGG Orthology (KO) as a controlled vocabulary. Bioinformatics 21:3787-3793

Mortazavi A, Williams BA, McCue K, Schaeffer L, Wold B (2008) Mapping and quantifying mammalian transcriptomes by RNASEq. Nat Methods 5:621-628

Pandey SP, Somssich IE (2009) The role of WRKY transcription factors in plant immunity. Plant Physiol 150:1648-1655

Pawlak-Sprada S, Arasimowicz-Jelonek M, Podgorska M, Deckert J (2011) Activation of phenylpropanoid pathway in legume plants exposed to heavy metals. Part I. Effects of cadmium and lead on 
phenylalanine ammonia-lyase gene expression, enzyme activity and lignin content. Acta Biochim Pol 58:211-216

Peng H, He X, Gao J, Ma H, Zhang Z, Shen Y, Pan G, Lin H (2015) Transcriptomic changes during maize roots development responsive to Cadmium (Cd) pollution using comparative RNAseq-based approach. Biochem Biophys Res Commun 464(4):1040-1047

Pereira GJG, Molina SMG, Lea PJ, Azevedo RA (2002) Activity of antioxidant enzymes in response to cadmium in Crotalaria juncea. Plant Soil 239:123-132

Phillips PC (2008) Epistasis-the essential role of gene interactions in the structure and evolution of genetic systems. Nat Rev Genet 9(11):855

Rother M, Krauss GJ, Grass G, Wesenberg D (2006) Sulphate assimilation under $\mathrm{Cd}^{2+}$ stress in Physcomitrella patens-combined transcript, enzyme and metabolite profiling. Plant Cell Environ 29:1801-1811

Santos ES, Abreu MM, Magalhães MCF (2016) Cistus ladanifer phytostabilizing soils contaminated with non-essential chemical elements. Ecol Eng 94:107-116

Sarwar N, Ishaq W, Farid G, Shaheen MR, Imran M, Geng M, Hussain S (2015) Zinc-cadmium interactions: impact on wheat physiology and mineral acquisition. Ecotoxicol Environ saf 122:528-536

Song WY, Park J, Eisenach C, Maeshima M, Lee Y, Martinoia E (2014) $\mathrm{ABC}$ transporters and heavy metals. In: Plant $\mathrm{ABC}$ transporters. Springer International Publishing, New York, pp 1-17

Thakur S, Singh L, Wahid ZA, Siddiqui MF, Atnaw SM, Din MF (2016) Plant-driven removal of heavy metals from soil: uptake, translocation, tolerance mechanism, challenges, and future perspectives. Environ Monit Assess 188:206

Tran TA, Popova LP (2013) Functions and toxicity of cadmium in plants: recent advances and future prospects. Turk $\mathbf{J}$ Bot 37(1):1-13

Wang Y, Xu L, Chen Y, Shen H, Gong Y, Limera C, Liu L (2013) Transcriptome profiling of radish (Raphanus sativus L.) root and identification of genes involved in response to lead $(\mathrm{Pb})$ stress with next generation sequencing. PLoS One 8:e66539

$\mathrm{Xu}$ Y (2016) Envirotyping for deciphering environmental impacts on crop plants. Theor Appl Genet 129:653-673

Zhang J, Duan GL (2008) Genotypic difference in arsenic and cadmium accumulation by rice seedlings grown in hydroponics. J Plant Nutr 31:2168-2182

Zheng N, Wang Q, Zhang X, Zheng D, Zhang Z, Zhang S (2007) Population health risk due to dietary intake of heavy metals in the industrial area of Huludao city, China. Sci Total Environ 387:96-104

Publisher's Note Springer Nature remains neutral with regard to jurisdictional claims in published maps and institutional affiliations. 